\title{
Meckel's diverticulum complications in three first-degree relatives: time to rethink familial predisposition?
}

\author{
loannis Patoulias ${ }^{1}$, Eleni Kogia ${ }^{1}$, Thomas Feidantsis ${ }^{1}$, Panagiota Michou ${ }^{2}$, Maria-Styliani Kalogirou ${ }^{3}$, \\ Spyridon Mitsoulis ${ }^{4}$, Dimitrios loannis Patoulias ${ }^{5}$ \\ ${ }^{1}$ First Department of Paediatric Surgery, General Hospital "G. Gennimatas", Aristotle University of Thessaloniki, Thessaloniki, \\ Greece \\ 2Department of Paediatrics, General Hospital "G. Gennimatas", Thessaloniki, Greece \\ ${ }^{3}$ Second Propaedeutic Department of Internal Medicine, General Hospital “Hippokration”, Aristotle University of Thessaloniki, \\ Thessaloniki, Greece \\ 4Department of Internal Medicine, General Hospital "G. Gennimatas", Thessaloniki, Greece \\ ${ }^{5}$ First Department of Internal Medicine, General Hospital "Hippokration", Thessaloniki, Greece
}

Gastroenterology Rev 2020; 15 (1): 80-81

DOI: https://doi.org/10.5114/pg.2020.93634

Address for correspondence: Dimitrios loannis Patoulias MD, First Department of Internal Medicine, General Hospital "Hippokration", 49 Konstantinoupoleos St, 54642 Thessaloniki, Greece, phone: +30 6946900777, e-mail: dipatoulias@gmail.com

Meckel's diverticulum represents a remnant of the omphalomesenteric duct, which results from the failure of the duct to obliterate during the $5^{\text {th }}-7^{\text {th }}$ gestational week [1]. It is identified in $0.3-2.9 \%$ of the general population, whereas complications develop in 4.2-9.0\% of the affected patients $[2,3]$. Tc ${ }^{99 m}$ scintigraphy constitutes the most widely used imaging modality, with sensitivity and specificity of $80-100 \%$ and $50-100 \%$, respectively [3].

After a systematic and comprehensive review of the relevant literature concerning the familial occurrence of Meckel's diverticulum in specific and omphalomesenteric duct remnants in general, we observed the extreme rarity of the presented case.

Herein, we present three cases of Meckel's diverticulum complications affecting three first-degree relatives (son, father, grandmother).

A male patient, 16 years old, was admitted to the Emergency Department due to periumbilical abdominal pain and recurrent episodes of vomiting during the last $24 \mathrm{~h}$. During physical examination, the presence of tenderness and positive rebound sign in the right lower quadrant of the abdomen were identified. Laboratory exams revealed the presence of polymorphonuclear leukocytosis and elevated C-reactive protein levels.

Therefore, after completion of preoperative evaluation, the patient underwent laparotomy under general endotracheal anaesthesia. After Lanz incision, ap- pendicectomy was performed. We then retracted the ileum to the surgical field, and we identified the presence of an inflamed Meckel's diverticulum with thick wall and loose adhesions to the adjacent mesentery, $3 \mathrm{~cm}$ in length, and with a base diameter of $1.4 \mathrm{~cm}$, found approximately $80 \mathrm{~cm}$ from the ileocecal valve (Figure 1). After opening the surgical specimen, we recognised the presence of erosions on the basis of the inflamed Meckel's diverticulum (Figure 2). Thus, we performed a sphenoid resection of the basis of Meckel's diverticulum and then an end-to-end anastomosis in two layers. Histopathological examination confirmed the diagnosis of inflammation of Meckel's diverticulum. The patient had an uncomplicated postoperative course and was discharged home in good general condition three days after surgery.

After thorough investigation of our patient's family history, we found that both his father and his grandmother, aged 41 and 68 years, respectively, had suffered in the past from Meckel's diverticulum complications. The father experienced gastrointestinal tract bleeding at the age of 2 years diagnosed with preoperative $T c^{99 m}$ scintigraphy. The grandmother underwent explorative laparotomy at the age of seven years in the context of acute abdomen diagnosis; inflammation of Meckel's diverticulum was documented. Therefore, appendicectomy and resection of Meckel's diverticulum were performed. 


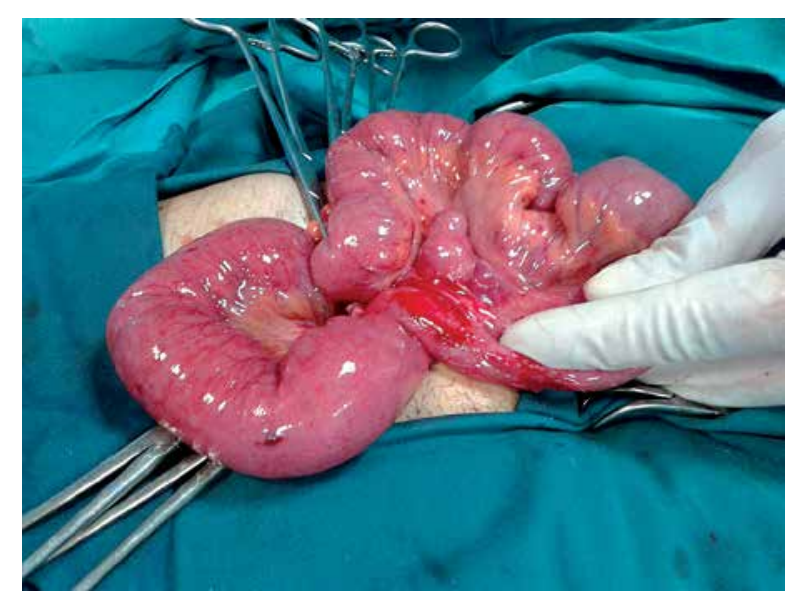

Figure 1. Meckel's diverticulum with macroscopic features of inflammation

Meckel's diverticulum is identified either in the context of complications development (obstruction, intussusception, diverticulitis, perforation, haemorrhage, neoplasia, fistula) or as an incident finding during exploratory laparotomy [2, 3].

According to Matsukuma et al., familial incidence of Meckel's diverticulum is $1 / 2500$ families [4]. After systematic and comprehensive research of the relevant literature, we found 15 cases of familial occurrence of Meckel's diverticulum, published between 1955 and 1994; however, a complication of Meckel's diverticulum was identified only in 5 cases [4-6].

Based on current knowledge, no familial predisposition for the presence of Meckel's diverticulum and its complications can be documented [2]. Through the present case report, we would like to shed further light on this interesting and unanswered issue. Future studies should focus on answering the following, remaining questions:

1. Is there a genetic background for the familial occurrence of Meckel's diverticulum and its complications?

2. What is the value of the presymptomatic diagnosis of Meckel's diverticulum presence in patients with positive familial history?

3. What is the value of targeted and prompt investigation for the presence of Meckel's diverticulum in patients with highly suspected symptomatology and positive familial history?

\section{Conflict of interest}

The authors declare no conflict of interest.

\section{References}

1. Sagar J, Kumar V, Shah DK. Meckel's diverticulum: a systematic review. J R Soc Med 2006; 99: 501-5.

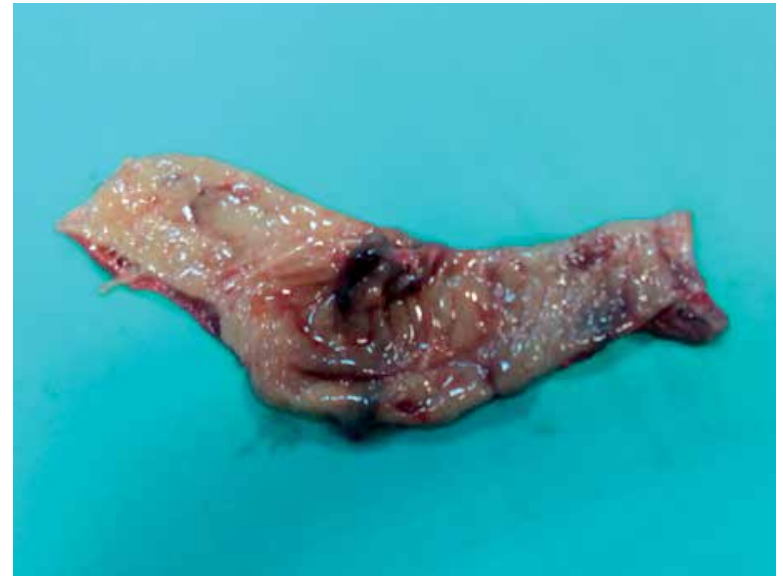

Figure 2. Presence of erosions at the base of Meckel's diverticulum

2. Pirzada U, Tariq H, Azam S, et al. A rare cause of abdominal pain in adults: Meckel's diverticulitis. Case Rep Gastroenterol 2018; 12: 709-14.

3. Hansen CC, Søreide K. Systematic review of epidemiology, presentation, and management of Meckel's diverticulum in the 21st century. Medicine 2018; 97: e12154.

4. Matsukuma Y, Matsuo Y, Sakaguchi M, et al. A case of siblings with Meckel's diverticulum diagnosed before operation. Acta Paediatr Jpn 1994; 36: 291-3.

5. Primrose JM, Henry RB. Symptomatic familial Meckel's diverticulum: two case reports and literature review. S D J Med 1977; 30: 7-9.

6. Lewenstein HJ, Levenson SS. Familial occurrence of Meckel's diverticulum. N Engl J Med 1963; 268: 311-2.

Received: 1.09 .2019

Accepted: 1.10 .2019 